\title{
Comportamiento productivo, degradación ruminal y producción de gas in vitro en ovinos alimentados con dietas a base de residuos pos-cosecha de Chenopodium quinoa
}

\author{
Productive Performance, RUMinal Degradation AND in VITRo gas PRODUCTION IN \\ SHEEP FED DIETS BASED ON POST-HARVEST RESIDUES OF Chenopodium quinoa
}

\author{
Oscar P. Núñez Torres ${ }^{1,2}$, Marcos-Barros Rodriguez ${ }^{1}$, Daniela Sanchez ${ }^{1}$, \\ Carlos Guishca-Cunuhay ${ }^{1}$
}

\section{Resumen}

El objetivo del estudio fue determinar el efecto de la ingesta de residuos pos-cosecha de Chenopodium quinoa sobre el comportamiento productivo, digestibilidad aparente de materia seca (MS), degradación ruminal de MS y producción de gas in vitro en ovinos. Se trabajó con un grupo de 12 carneros de 6 meses para medir la ganancia de peso y consumo voluntario durante 75 días (15 días de adaptación) y la digestibilidad in vivo en jaulas metabólicas. Un segundo grupo de 6 ovinos mayores de 8 meses y canulados para el estudio de degradación ruminal in situ de MS mediante la técnica de la bolsa de nylon y para la producción de gas in vitro. Se utilizó un diseño aleatorizado con dos tratamientos: T1: (95.8\% Pennisetum clandestinum), T2: (75.8\% P. clandestinum y 20\% afrecho de C. quinoa). El consumo voluntario en T2 fue mayor que en T1, pero el consumo voluntario por $\mathrm{kg} \mathrm{PV}{ }^{0.75}$ no mostró diferencia significativa entre tratamientos. La conversión alimenticia fue mejor en T2 frente a T1 (7:91 a 9:29). La ganancia de peso fue superior en T2. La digestibilidad aparente de la MS no mostró diferencias entre tratamientos. La producción de gas in vitro fue menor en el T2 con respecto a T1. Los parámetros de degradación ruminal no presentaron diferencias entre tratamientos. Con respecto a la tasa de degradación (c) en porcentaje por hora no se observó diferencias entre forrajes. Se concluye que los rastrojos de forraje de $C$. quinoa pueden ser incluidos en un $20 \%$ en la dieta de los ovinos para ganancias moderadas de peso vivo al usar pastos de baja calidad nutricional como $P$. clandestinum.

Palabras clave: digestibilidad; producción de gas; in vitro; in situ; consumo voluntario; ovejas

\footnotetext{
${ }^{1}$ Facultad de Ciencias Agropecuarias, Universidad Técnica de Ambato, Cevallos, Tungurahua, Ecuador

${ }^{2}$ E-mail: op.nunez@uta.edu.ec
}

Recibido: 23 de enero de 2018

Aceptado para publicación: 27 de junio de 2018 
The aim of the study was to determine the effect of the post-harvest residue intake of Chenopodium quinoa on the productive performance, apparent digestibility of dry matter (DM), ruminal degradation of DM and in vitro gas production in sheep. A group of 12 rams of 6 months were used to evaluate body weight gain and voluntary consumption for 75 days ( 15 days of adaptation) and in vivo digestibility in metabolic cages. A second group of 6 sheep over 8 months of age and rumen cannulated were used for the study of in situ ruminal degradation of DM using the nylon bag technique and for in vitro gas production. A randomized design with two treatments was used: T1: (95.8\% Pennisetum clandestinum), T2: (75.8\% P. clandestinum and $20 \%$ bran of C. quinoa). Voluntary intake in $\mathrm{T} 2$ was higher than in $\mathrm{T} 1$, but voluntary intake per $\mathrm{kg} \mathrm{PV}^{0.75}$ showed no significant difference between treatments. The feed conversion was better in T2 versus T1 (7:91 to 9:29). The weight gain was higher in T2. The apparent digestibility of the DM did not show differences between treatments. In vitro gas production was lower in T2 with respect to $\mathrm{T} 1$. The parameters of ruminal degradation did not show differences between treatments. With respect to the rate of degradation (c) in percentage per hour no differences were observed between forages. It is concluded that forage residues of $C$. quinoa can be included in $20 \%$ of the diet of sheep for moderate body weight gain when using low nutritional quality pastures such as $P$. clandestinum.

Key words: digestibility; gas production; in vitro; in situ; voluntary intake; sheep

\section{INTRODUCCIÓN}

La producción de rumiantes en el altiplano andino del Ecuador se caracteriza por el predominio de monocultivos de gramíneas que afectan el rendimiento productivo de los animales (Barros-Rodriguez et al., 2017a), debido al elevado contenido de fibra y lignina que estas plantas aportan a la dieta que generan pérdidas de energía en forma de gases de efecto invernadero $\left(\mathrm{CO}_{2}, \mathrm{CH}_{4}\right)$ del 8-12\% (Kurihara et al., 1999). De estos, es de importancia el $\mathrm{CH}_{4}$ debido a que aporta alrededor del $18 \%$ del calentamiento global generado por las explotaciones pecuarias (Carmona et al., 2005).

Como alternativa para subsanar esta problemática se plantea la incorporación de los residuos de la cosecha de cultivos agrícolas en la dieta de rumiantes, debido a los beneficios que tienen sobre la fermentación ruminal, enfocados a optimizar la productividad de los animales y a disminuir la genera- ción de gases de efecto invernadero. Entre estos residuos se encuentra la quinoa (Chenopodium quinoa), el chocho (Lupinus mutabilis) y el amaranto (Amaranthus cruentus), caracterizados por su alto valor nutritivo y presencia de compuestos secundarios (Barros-Rodríguez et al., 2017b). Así, Cantú et al. (2000) evaluaron cultivares de C. quinoa en etapa de panoja a floración, determinando un contenido de proteína cruda de $17.6 \%$ en la etapa inicial y de $10-18.9 \%$ en grano. Así mismo, Vélez-Terranova et al. (2014) resaltan los posibles beneficios por compuestos secundarios como la saponina. De esta forma, la importancia del uso de este subproducto radica en los volúmenes producidos que viabilizan su utilización y que no generan controversia social, ya que no compiten con su empleo en la alimentación humana.

Con base en estos antecedentes, el objetivo de esta investigación fue determinar el efecto de la ingestión de residuos pos-cosecha de Chenopodium quinoa sobre el 
comportamiento productivo, digestibilidad aparente de la materia seca (MS), la degradación ruminal de la MS y producción de gas in vitro en ovinos.

\section{MATERIALES y Métodos}

\section{Animales y Dietas}

La investigación se llevó a cabo en la Facultad de Ciencias Agropecuarias de la Universidad Técnica de Ambato, ubicada en el Cantón Cevallos, provincia de Tungurahua, Ecuador, a una altitud de $2890 \mathrm{~m}$. En el sector se tienen temperaturas máximas de $20^{\circ} \mathrm{C}$ y mínimas de $7{ }^{\circ} \mathrm{C}$ y una temperatura ambiente promedio de $15{ }^{\circ} \mathrm{C}$.

Se trabajo con un cultivo de quinoa $(C$. quinoa) sembrada como fuente de grano para el consumo humano y una pastura de kikuyo (Pennisetum clandestinum). El residuo pos-cosecha de $C$. quinoa se obtuvo inmediatamente después de su trillado (separación del grano de la planta seca), siendo procesada en un molino a martillo (Cremasco DP2, Brasil) a $2 \mathrm{~mm}$ de tamaño de partícula. Además, se recolectó $30 \mathrm{~kg}$ de materia fresca de $P$. clandestinum cortado a una edad de 35 días, que fue secada mediante exposición al sol y molida posteriormente. Los forrajes molidos fueron incorporados en una mezcla integral, según se muestra en el Cuadro 1.

La materia seca (MS) (\# 7.007) y ceniza (\# 7.009) se determinaron según la AOAC (1990). La fibra detergente neutro (FDN) y fibra detergente ácida (FDA) se determinaron mediante el método 12 y 13 respectivamente, ANKOM2000 analizador de fibra (ANKOM Technology, Macedon, NY, EEUU). La PC se determinó por análisis elemental $(\mathrm{N})$ utilizando un LECO CHN 628 (LECO Corporation).
Se usaron 18 ovinos machos criollos en dos grupos experimentales. El primero conformado por 12 ovinos de 6 meses de edad y peso vivo promedio de $20.9 \pm 1.0 \mathrm{~kg}$. El segundo grupo fue con seis ovinos mayores a 8 meses, con peso vivo promedio de $28.9 \pm 1.0$ $\mathrm{kg}$ y provistos de cánulas ruminales. La investigación se desarrolló en dos fases: a) de campo (parámetros productivos, digestibilidad in vivo, degradación ruminal) y b) en laboratorio (producción de gas in vitro).

Los animales fueron alojados en un galpón con jaulas individuales de $1.8 \times 2.0 \mathrm{~m}$, provistas con comederos y bebederos plásticos. Para medir la digestibilidad in vivo se alojaron los 12 ovinos en jaulas metabólicas, las cuales contaban con un sistema de recolección de heces y orina, además de los recipientes para el agua y el alimento. Para la degradación in vivo los animales permanecieron en grupos de tres animales por jaula $(1.8 \times 4.9 \mathrm{~m})$.

Cuadro 1. Inclusión de niveles de residuos pos-cosecha de Chenopodium quinoa y composición química de las dietas experimentales $(\mathrm{g} / \mathrm{kg} \mathrm{MS})$

\begin{tabular}{lcc}
\hline & \multicolumn{2}{c}{ Tratamientos } \\
\cline { 2 - 3 } Ingredientes & $\mathrm{T} 1$ & $\mathrm{~T} 2$ \\
\hline P. clandestinum & 95.8 & 75.8 \\
C. quinoa & 0 & 20 \\
Sal mineral & 0.20 & 0.20 \\
Melaza & 4 & 4 \\
\hline Total & 100 & 100 \\
\hline Composición química $(\%)$ & \\
Materia seca & 88.0 & 88.4 \\
Materia orgánica & 83.4 & 86.8 \\
Proteína cruda & 7.9 & 6.7 \\
Fibra detergente & 65.0 & 64.8 \\
neutro & & \\
Fibra detergente & 31.42 & 34.48 \\
ácida & 16.5 & 13.1 \\
Cenizas &
\end{tabular}


La alimentación fue a base de una mezcla forrajera de $P$. clandestinum y residuos pos-cosecha de $C$. quinua suministrado en base seca en una dieta integral (Cuadro 1). El alimento y el agua fueron ofrecidos $a d$ libitum. El experimento tuvo una duración de 75 días: 15 de adaptación y 60 días de muestreo para la prueba de comportamiento productivo. Para la digestibilidad in vivo tuvo un periodo de 15 días, con 5 días de adaptación y 10 días de muestreo y para la degradación in situ tuvo una duración de 17 días, con periodos de 96 horas por tratamiento y sus repeticiones.

\section{Comportamiento Productivo}

El consumo de alimento se estimó mediante método directo (alimento ofrecido alimento rechazado), pesando el alimento ofrecido y el rechazado después de $24 \mathrm{~h}$, con una balanza digital de $5 \mathrm{~kg}$ de capacidad, por cinco días consecutivos. Este procedimiento se realizó cada 15 días.

La ganancia de peso se estimó mediante método directo (peso inicial - peso final). Los animales fueron pesados cada 15 días, con 14 horas de ayuno previo, utilizando una balanza digital (50 kg de capacidad).

La conversión alimenticia se obtuvo mediante estimación matemática entre la relación de alimento consumido en gramos y la ganancia de peso. Conversión alimenticia $=$ consumo de alimento / ganancia de peso.

\section{Digestibilidad Aparente de MS in vivo}

Se estimó mediante el método directo en jaulas metabólicas, pesando el alimento ofrecido y restando el peso de las heces. Este procedimiento se hizo después de los 60 días de muestreo para la determinación del comportamiento productivo. En el laboratorio se utilizó una estufa para conseguir el porcentaje de MS de las muestras de pastos, así como de las raciones y las heces de los tratamientos en los respectivos muestreos.

\section{Degradación Ruminal in situ}

Se evaluó mediante la técnica de la bolsa de nylon en el rumen, descrita por Ørskov et al. (1980). En cada animal se colocó una bolsa con $3 \mathrm{~g}$ de MS de cada tratamiento y se incubaron por $0,4,8,12,24,36,48,72 \mathrm{y}$ $96 \mathrm{~h}$. Las bolsas fueron removidas al final de los periodos de incubación, lavadas con agua corriente y secadas a $60^{\circ} \mathrm{C}$. Las bolsas empleadas para medir la pérdida por lavado $(0$ h) no se incubaron en el rumen y solo se lavaron con agua corriente. Los residuos se almacenaron en bolsas de polietileno a $-4^{\circ} \mathrm{C}$. La desaparición de los nutrientes fue calculada como una proporción del material incubado y residual. Los datos fueron ajustados a la ecuación: $\mathrm{Y}=\mathrm{a}+\mathrm{b}\left(1-\mathrm{e}^{-\mathrm{ct}}\right)$ (Ørskov y McDonald, 1979).

\section{Producción de Gas in vitro}

Se obtuvo el contenido ruminal (líquido y fracciones solidas) por separado de cada individuo (tres animales por tratamiento). El contenido ruminal se recolectó antes de alimentar a los animales y se mantuvo a $39{ }^{\circ} \mathrm{C}$ en un recipiente de plástico sellado durante el transporte al laboratorio. El análisis se realizó dentro de la hora de recolección en un medio rico en nitrógeno, según Menke y Steingass (1988).

La producción de gas se determinó según la metodología descrita por Theodorou et al. (1994). Para esto, $0.5 \mathrm{~g}$ de MS de cada tratamiento se colocó en botellas de vidrio de $100 \mathrm{ml}$ de capacidad nominal. Se añadió 60 $\mathrm{ml}$ de inóculo ruminal (70:30 medio/inóculo ruminal) bajo un flujo de $\mathrm{CO}_{2}$ constante. Las botellas se sellaron y se incubaron a $39-40{ }^{\circ} \mathrm{C}$. La presión de gas y el volumen se midieron manualmente a las 3, 6, 9, 12, 18, 24, 36 y 48 $\mathrm{h}$ de la incubación con un transductor de presión Delta OHM modelo DO 9704 (Delta OHM, Padova, Italia) y jeringas plásticas. Para cada tratamiento se utilizaron seis botellas (repeticiones) por cada tiempo de incubación y tres botellas adicionales como 
blanco. El total de producción de gas fue estimada por $0.5 \mathrm{~g}$ de MS fermentable.

\section{Análisis Estadístico}

Se utilizo un diseño completamente al azar con dos tratamientos y seis repeticiones. Los datos de consumo voluntario, ganancia de peso, digestibilidad aparente de la MS y producción de gas in vitro se analizaron utilizando el PROC GLM del SAS (2009) y la comparación de medias mediante la prueba de Tukey. La degradación ruminal de la MS se analizó con el programa Graphpad Prism 6 (San Diego, EEUU).

\section{Resultados}

El consumo voluntario de los ovinos alimentados con $P$. clandestinum + afrecho de C. quinoa (T2) fue $152 \mathrm{~g} \mathrm{MS/día} \mathrm{mayor} \mathrm{que}$ con la dieta de $100 \%$ P. clandestinum (T1) (Cuadro 2; $\mathrm{p}=0.0428$ ). En forma similar, el consumo voluntario de materia orgánica (MO) fue mayor $(\mathrm{p}=0.0001)$ en T2 frente a T1. El consumo voluntario de proteína cruda $(\mathrm{PC})$ mostró una ligera diferencia $(\mathrm{p}=0.5591)$ en- tre tratamientos. El consumo voluntario por kg $\mathrm{PV}^{0.75}$ no mostró diferencia significativa $(\mathrm{p}=0.1385)$ entre tratamientos.

La mejor conversión alimenticia fue para T2 con respecto a T1 (7:91 a 9:29, respectivamente $(\mathrm{p}=0.0178)$, al igual que la ganancia de peso (T2 con $29.35 \mathrm{~g} / \mathrm{d}$ más que T1). La digestibilidad aparente de la MS no presentó diferencias significativas entre tratamientos $(\mathrm{p}=0.2689)$. La producción de gas in vitro (ml/0.5 g MS fermentable) fue menor para $\mathrm{T} 2(\mathrm{p}=0.0215)$ (Cuadro 3).

Las dietas experimentales no mostraron diferencias entre tratamientos para los parámetros de degradación ruminal (Cuadro 4). Con respecto a la degradación de la MS de los forrajes (kikuyo y quinua), la fracción soluble (a) muestra diferencias $(p<0.05)$ entre forrajes, siendo el de mayor porcentaje para kikuyo (30\%). La fracción insoluble, pero potencialmente degradable (b) muestra diferencias $(p<0.05)$ entre forrajes con el mayor porcentaje de degradación para kikuyo (44\%). Con respecto a la tasa de degradación (c) en porcentaje por hora, no se observó diferencias significativas entre forrajes.

Cuadro 2. Consumo voluntario de materia seca (MS), materia orgánica (MO), proteína cruda (PC) y $\mathrm{kg} \mathrm{PV}^{0.75}$ (g/kg MS excepto donde se menciona lo contrario)

\begin{tabular}{lcccc}
\hline & \multicolumn{2}{c}{ Tratamientos } & \multirow{2}{*}{ EEM } & p \\
\cline { 2 - 3 } & T1 & T2 & & 0.0463 \\
CV de MS (kg/kg MS) & $0.594^{\mathrm{b}}$ & $0.746^{\mathrm{a}}$ & & 0.0428 \\
CVMO & $496.3^{\mathrm{b}}$ & $644.5^{\mathrm{a}}$ & 40.32 & 0.0001 \\
CVPC & 47.0 & 49.9 & 3.45 & 0.5591 \\
CV kg PV $^{0.75}$ & 59.02 & 69.41 & 4.562 & 0.1385 \\
\hline
\end{tabular}

ab Medias con letras distintas dentro de filas difieren significativamente $(p<0.05)$

EEM: error estándar de la media

$\mathrm{CV}$ : consumo voluntario

CVMO: consumo voluntario de materia orgánica

CVPC: consumo voluntario de proteína cruda

$\mathrm{PV}^{0.75}$ : Peso vivo metabólico

T1: $95.8 \%$ P. clandestinum; T2: $75.8 \%$ P. clandestinum $+20 \%$ C. quinoa 
Cuadro 3. Ganancia de peso, conversión alimenticia, digestibilidad aparente de la materia seca (MS) y producción de gas in vitro ( $\mathrm{g} / \mathrm{kg}$ MS excepto donde se menciona lo contrario)

\begin{tabular}{|c|c|c|c|c|}
\hline & \multicolumn{2}{|c|}{ Tratamientos } & \multirow{2}{*}{ EEM } & \multirow{2}{*}{$\mathrm{p}$} \\
\hline & $\mathrm{T} 1$ & $\mathrm{~T} 2$ & & \\
\hline Ganancia de peso(g/d) & $64.48^{\mathrm{b}}$ & $93.83^{\mathrm{a}}$ & 2.662 & 0.0001 \\
\hline $\mathrm{CA}$ & $9.29^{\mathrm{b}}$ & $7.91^{\mathrm{a}}$ & 0.637 & 0.0178 \\
\hline DAMS (g/kg MS) & 441.46 & 464.63 & 13.978 & 0.2689 \\
\hline PGIV $0.500 \mathrm{~g} / \mathrm{MSFM}$ & $267.09^{\mathrm{a}}$ & $227.26^{\mathrm{b}}$ & 10.346 & 0.0215 \\
\hline
\end{tabular}

${ }^{a b}$ Medias con letras distintas dentro de filas difieren significativamente $(p<0.05)$

EEM: error estándar de la media

CA: conversión alimenticia

DAMS: digestibilidad aparente de la materia seca

PGIV: producción de gas in vitro

MSFM: materia seca fermentable

T1: $95.8 \%$ P. clandestinum; T2: 75.8\% P. clandestinum + 20\% C. quinoa

Cuadro 4. Parámetros de degradación ruminal de los tratamientos y rastrojo de Chenopodium quinoa y Pennisetum clandestinum (g/kgMS)

\begin{tabular}{|c|c|c|c|}
\hline \multirow{2}{*}{$\begin{array}{l}\text { Parámetros de } \\
\text { degradación }\end{array}$} & \multicolumn{2}{|c|}{ Tratamientos } & \multirow{2}{*}{$\mathrm{p}$} \\
\hline & $\mathrm{T} 1$ & $\mathrm{~T} 2$ & \\
\hline $\mathrm{T}_{0}$ & $380.0 \pm 17.00$ & $352.7 \pm 13.85$ & \\
\hline A & $373.2 \pm 13.50$ & $370.5 \pm 13.45$ & $>0.05$ \\
\hline $\mathrm{B}$ & $577.7 \pm 179.0$ & $474.3 \pm 52.96$ & $>0.05$ \\
\hline$a+b$ & 95.09 & 84.48 & \\
\hline $\mathrm{C}$ & $0.008 \pm 0.0042$ & $0.015 \pm 0.0037$ & $>0.05$ \\
\hline \multirow[t]{3}{*}{$r^{2}$} & 0.88 & 0.92 & \\
\hline & \multicolumn{2}{|c|}{ Forrajes } & \\
\hline & C. quinoa & P. clandestinum & \\
\hline $\mathrm{T}_{0}$ & $215.5 \pm 4.50$ & $321.3 \pm 37.97$ & \\
\hline $\mathrm{A}$ & $181.1 \pm 17.25 b$ & $304.9 \pm 25.52 \mathrm{a}$ & $<0.05$ \\
\hline $\mathrm{B}$ & $285.7 \pm 17.90 \mathrm{~b}$ & $445.1 \pm 93.98 a$ & $<0.05$ \\
\hline$a+b$ & 46.68 & 75.0 & \\
\hline $\mathrm{C}$ & $0.034 \pm 0.0070$ & $0.015 \pm 0.0075$ & $>0.05$ \\
\hline$r^{2}$ & 0.85 & 0.74 & \\
\hline
\end{tabular}

${ }^{\mathrm{ab}}$ Medias con letras distintas dentro de columnas difieren significativamente $(p<0.05)$

$T_{0}$ : tiempo cero (muestras lavadas en laboratorio)

a: fracción soluble, b: fracción insoluble pero potencialmente degradable, c: tasa de degradación

(porcentaje por hora), To: tiempo cero, a+b: potencial degradación (en porcentaje)

T1: $95.8 \%$ P. clandestinum; T2: $75.8 \%$ P. clandestinum $+20 \%$ C. quinoa 


\section{Discusión}

El consumo voluntario de nutrientes fue mayor al adicionar rastrojo de C. quinoa, probablemente debido a sus características físicoquímicas que permiten mejorar el consumo, el cual está influenciado por el sabor, olor, textura y apariencia del alimento (Allison, 1985). Esta dieta favorece una mejor estimulación sensorial y con ello una mayor preferencia por el alimento (Grovum y Chapman, 1988), resultados que coinciden con los reportados por Kaitho et al. (1997).

La mayor ganancia de peso observada en este estudio estuvo relacionada al mayor consumo voluntario de nutrientes (Cuadro 4) (Mejía, 2002) y posiblemente a la menor producción de gas en los animales de T2 (Cuadro 3), lo cual mejora la utilización de la energía por parte del animal (Barros-Rodríguez et al., 2014a).

La digestibilidad aparente de la MS no muestra diferencia significativa entre tratamientos debido al contenido de fibra en las dietas (Cuadro 3) (Arelovich et al., 2008); sin embargo, el coeficiente de digestibilidad obtenido es superior a los reportados por Sánchez y Zambrano (2007), quienes evaluaron subproductos de cosechas (pacas de maíz, arroz y soya) enriquecidos con melaza y urea obteniendo entre 36.7 y $41.6 \%$ de digestibilidad y $4.5 \mathrm{~kg}$ de ganancia de peso frente al $46.5 \%$ de coeficiente de digestibilidad y $5.6 \mathrm{~kg}$ de peso con la inclusión de subproducto de C. quinoa en esta investigación. Esta mejora puede atribuirse a los compuestos secundarios que aumentan la digestibilidad de la dieta (Barros-Rodríguez et al., 2014b) y posiblemente a componentes estructurales de la fibra de la quinua que aportan mayor biodisponibilidad (Barahona y Sánchez, 2005). Esto favorece el consumo voluntario, menor tiempo de retención ruminal y mayor tasa de pasaje (Torres et al., 2009).
La menor producción de gas in vitro observada en $\mathrm{T} 2$ se debe probablemente a compuestos secundarios como las saponinas que tienen potencial para mejorar el flujo de proteína microbial y disminuir la metanogénesis (Goel y Makkar, 2012). Así, Bonilla y Lemus (2012) manifiestan que al usar un $0.012 \%$ de saponinas se disminuye el $\mathrm{CH}_{4}$ por efecto anti-protozoarios más la capacidad de desviar iones hidrogeno de organismos metanogénicos. Además, se ha observado una disminución del tiempo de retención ruminal y una menor digestibilidad de la fibra (Galindo et al., 2014).

A pesar de no haberse encontrado diferencia significativa entre tratamientos, se observa que la tasa de degradación más alta se obtuvo en T2, probablemente por las características del alimento y su contenido de carbohidratos no estructurales (Salem et al., 2011) coadyuvado al potencial de estos compuestos secundarios para modificar la velocidad de degradación y, por lo tanto, el pasaje de los nutrientes a través del tracto gastrointestinal debido a una inhibición de ciertas bacterias y protozoarios ruminales (Galindo et al., 2014).

\section{Conclusiones}

El uso de rastrojos de Chenopodium quinoa en un $20 \%$ en la dieta para ovinos se puede mejorar los parámetros productivos, posiblemente por una mejora del ambiente ruminal generando una mayor producción de proteína microbiana, coadyuvado por un mejor aprovechamiento de la energía debido a una menor producción de gas.

\section{Literatura Citada}

1. Allison CD. 1985. Factors affecting forage intake by ruminants: a review. J Range Manage 38: 305-311. doi: $10.2307 / 3899409$ 
2. Arelovich HM, Abney CS, Vizcarra A, Galyean ML. 2008. Effects of dietary neutral detergent fiber on intakes of dry matter and net energy by dairy and beef cattle: analysis of published data. Prof Anim Scientist 24: 375-383. doi: 10.15232/S1080-7446(15)30882-2

3. Barahona R, Sánchez S. 2005. Limitaciones físicas y químicas de la digestibilidad de pastos tropicales y estrategias para aumentarla. Corpoica 6: 69-82. doi: 10.21930/rcta.vol6_num1_art:39

4. Barros-Rodríguez M, Sandoval-Castro CA, Solorio-Sánchez J, Sarmiento-Franco LA, Rojas-Herrera $R$, Klieve AV. 2014a. Leucaena leucocephala in ruminant nutrition. Trop Subtrop Agroecosyst 17: 173-183.

5. Barros-Rodríguez MA, SolorioSánchez FJ, Sandoval-Castro CA, Ahmed AMM, Rojas-Herrera R, Briceño-Poot EG, Ku-Vera JC. 2014b. Effect of intake of diets containing tannins and saponins on in vitro gas production and sheep performance. Anim Prod Sci 54: 1486-1489. doi: 10.1071/ AN14294

6. Barros-Rodríguez, M, Rovalino-Núñez V, Núñez-Torres O, Mera-Andrade R, Artieda-Rojas J, Vaca-Freire L, et al. 2017a. Composición química, cinética de degradación ruminal y producción de gas in vitro de arvenses con potencial forrajero. Livest Res Rural Dev 29(4). [Internet]. Disponible en: http:// www.lrrd.org/lrrd29/4/barr29071.html

7. Barros-Rodríguez M, Oña-Rodríguez J, Mera-Andrade R, Artieda-Rojas J, Curay-Quispe S, Aviles-Esquivel D, Solorio-Sánchez J, et al. 2017b. Degradación ruminal de dietas a base de biomasa pos-cosecha de Amaranthus cruentus: efecto sobre los protozoos del rumen y producción de gas in vitro. Rev Inv Vet Perú 28: 812-821. doi: 10.15381/ rivep.v28i4.13931

8. Bonilla JA, Lemus C. 2012. Emisión de metano entérico por rumiantes y su contribución al calentamiento global y al cambio climático: revisión. Rev Mex Cienc Pecu 3: 215-246.

9. Cantú DJ, Guerrero JBS, García RR, Sánchez JLA. 2000. Quinua para forraje: análisis de concentración y composición de saponinas. [Internet]. Disponible en: http://www.uaaan.mx/DirInv/ Avances_2002/Oleag/JassoQuinua\%20.pdf

10. Carmona JC, Bolívar DM, Giraldo $L A .2005$. El gas metano en la producción ganadera y alternativas para medir sus emisiones y aminorar su impacto a nivel ambiental y productivo. Rev Colomb Cienc Pec 18: 49-63.

11. Galindo J, González N, Marrero Y, Sosa A, Ruiz, T, Febles G, Torres V, et al. 2014. Effect of tropical plant foliage on the control of methane production and in vitro ruminal protozoa population. Cuban J Agr Sci 48: 359-364.

12. Goel G, Makkar HPS. 2012. Methane mitigation from ruminants using tannins and saponins. Trop Anim Health Pro 44: 729739. doi: 10.1007/s11250-011-9966-2

13. Grovum WL, Chapman HW. 1988. Factors affecting the voluntary intake of food by sheep. 4. The effects of additives representing the primary tastes on sham intakes by oesophagealfistulated sheep. Brit J Nutr 59: 63-72. doi: 10.1079/BJN19880010

14. Kaitho RJ, Umunna NN, Nsahlai IV, Tamminga S, Van Bruchem T, Hanson I. 1997. Palatability of wilted and dried multipurpose tree species fed to sheep and goats. Anim Feed Sci Tech 65: 151-165. doi: 10.1016/S0377-8401(96)01092-9

15. Kurihara M, Magner T, Hunter RA, McCrabb GJ. 1999. Methane production and energy partition of cattle in the tropics. Brit J Nutr 81: 227-234. doi: $10.1017 / \mathrm{S} 0007114599000422$

16. Mejía J. 2002. Consumo voluntario de forraje por rumiantes en pastoreo. Acta Univ 12: 56-63.

17. Menke KH, Steingass H. 1988. Estimation of the energetic feed value obtained from chemical analysis and in vitro gas production using rumen fluid. Anim Res Develop 28: 7-55. 
18. Orskov E, McDonald I. 1979. The estimation of protein degradability in the rumen from incubation measurements weighted according to rate of passage. J Agric Sci 92: 499-503. doi: 10.1017/ S0021859600063048

19. Orskov ER, Hovell FDB, Mould F. 1980. The use of the nylon bag technique for the evaluation of feedstuffs. Trop Anim Prod 5: 195-213.

20. Salem AFZ, El-Adawy M, Gado H, Camacho LM, Ronquillo M, Alsersy H, Borhami BE. 2011. Effects of exogenous enzymes on nutrients digestibility and growth performance in sheep and goats. Trop Subtrop Agroecosyst 14: 867-874.

21. Sánchez LA, Zambrano GD. 2007. Valoración nutritiva de los principales subproductos agrícolas para la alimentación de ovinos tropicales en la parte alta de la cuenca del río Guayas. Univ.
Técnica Estatal de Quevedo. Ecuador. [Internet]. Disponible en: http:// repositorio.educacionsuperior.gob.ec/ bitstream/28000/781/1/L-SENESCYT0083.pdf

22. Theodorou M, Williams B, Dhanoa M, Mcallan A, France J. 1994. A simple gas production method using a pressure transducer to determine the fermentation kinetics of ruminant feeds. Anim Feed Sci Tech 48: 185-197. doi: 10.1016/03778401(94)90171-6

23. Torres G, Arbaiza T, Carcelén F, Lucas O. 2009. Comparación de las técnicas in situ, in vitro y enzimática (celulasa) para estimar la digestibilidad de forrajes en ovinos. Rev Inv Vet Perú 20: 5-9. doi: 10.15381/rivep.v20i1.348

24. Vélez-Terranova M, Campos Gaona R, Sánchez-Guerrero H. 2014. Uso de metabolitos secundarios de las plantas para reducir la metanogénesis ruminal. Trop Subtrop Agroecosyst 17: 489-499. 